\title{
Isolamento social em tempos de pandemia do COVID-19 e sua relação com a obesidade
}

\author{
Social isolation in times of the COVID-19 pandemic and its relationship to obesity
}

Aislamiento social en tiempos de la pandemia de COVID-19 y su relación con la obesidad

João Victor Machado Cristo

ORCID: https://orcid.org/0000-0001-7784-8892

Universidade Federal do Pará, Brasil

E-mail: victorcristo2019@gmail.com

Filipe Santos da Silva

ORCID: https://orcid.org/0000-0003-3461-5698

Universidade Federal do Pará, Brasil

E-mail: filipe.silva@ics.ufpa.br

Manuela Salame Serique

ORCID: https://orcid.org/0000-0002-0220-7254

Centro Universitário do Estado Pará, Brasi

E-mail: manuserique@gmail.com

Micaella Yanne Fender Lobato

ORCID: https://orcid.org/0000-0001-9707-6535

Universidade Federal do Pará, Brasil

E-mail: micaellafender@gmail.com

Fernanda Protázio Silva

ORCID: https://orcid.org/0000-0003-0696-8138 Universidade Federal do Pará, Brasil

E-mail: fernanda.protazios13@gmail.com

Fernanda de Souza Parente

ORCID: https://orcid.org/0000-0003-3831-6011 Universidade Federal do Pará, Brasil E-mail: feesp7@gmail.com

Amanda de Paula

ORCID: https://orcid.org/0000-0001-5300-1961 Universidade do Estado do Pará, Brasil

E-mail: amandadepaularoraima@gmail.com

Davi Gonçalves Campos

ORCID: https://orcid.org/0000-0002-7096-5436

Centro Universitário do Estado Pará, Brasil E-mail: davi.gol10campos@gmail.com

Cristal Louise Antunes Gonçalves ORCID: https://orcid.org/0000-0003-4490-7088 Universidade Federal do Pará, Brasil E-mail: cristalgoncalves@gmail.com

Beatriz Sayuri Vieira Ishigaki

ORCID: https://orcid.org/0000-0001-6346-6025 Universidade do Estado do Pará, Brasil E-mail: beatrizishigaki00@gmail.com

Lorena Soriano de Melo Lima

ORCID: https://orcid.org/0000-0002-7056-1298

Centro Universitário do Estado Pará, Brasi

E-mail: lorenamelolima@ hotmail.com

Santino Carvalho Franco

ORCID: https://orcid.org/0000-0001-9412-7632 Universidade do Estado do Pará, Brasi

E-mail: santinomed@yahoo.com.br

\section{Resumo}

Introdução: A obesidade é uma doença crônica, multifatorial e que gera repercussões sistêmicas; caracterizada por um Índice de Massa Corporal (IMC) maior que 30. O isolamento social na pandemia do COVID-19 interferiu diretamente no tratamento dos pacientes obesos e adoeceu pacientes antes sem a comorbidade. Metodologia: Trata-se de uma revisão bibliográfica qualitativa, com busca de estudos nas bases de dados Biblioteca Virtual em Saúde (BVS), Google Scholar, PubMed e Scientific Library Online (SciELO). Para a pesquisa, utilizou-se os descritores COVID-19, isolamento social, obesidade, saúde mental e transtornos alimentares. Incluíram-se publicações entre 2017 e 2021 em 
português e inglês disponíveis em texto completo. Foram selecionados 15 artigos dos 71 encontrados. Resultados $e$ discussão: percebeu-se que o isolamento interfere negativamente no tratamento de pacientes obesos, pois os locais de prática de exercícios físicos ficaram restritos e a reclusão domiciliar dificulta o tratamento. Outro problema está no desenvolvimento de compulsão alimentar, já que a comida está sendo utilizada para aliviar problemas de ansiedade, depressão e estresse. Conclusão: O COVID-19 causou problemas que vão além dos casos de infecção pelo vírus, pois o isolamento social afetou diretamente o tratamento de pacientes obesos.

Palavras-chave: COVID-19; Obesidade; Isolamento social.

\begin{abstract}
Introduction: Obesity is a chronic, multifactorial disease that generates systemic repercussions; characterized by a Body Mass Index (BMI) greater than 30. Social isolation in the COVID-19 pandemic directly interfered in the treatment of obese patients and sickened patients before without comorbidity. Methodology: This is a qualitative literature review, with a search for studies in the Virtual Health Library (VHL), Google Scholar, PubMed and Scientific Library Online (SciELO) databases. For the research, the descriptors COVID-19, social isolation, obesity, mental health and eating disorders were used. Publications between 2017 and 2021 in Portuguese and English available in full text were included. 15 articles were selected from the 71 found. Results and discussion: it was noticed that isolation interferes negatively in the treatment of obese patients, as places for physical exercise were restricted and home confinement makes treatment difficult. Another problem is the development of binge eating, as food is being used to relieve anxiety, depression and stress problems. Conclusion: COVID-19 caused problems that go beyond cases of virus infection, as social isolation directly affected the treatment of obese patients.
\end{abstract}

Keywords: COVID-19; Obesity; Social isolation.

\title{
Resumen
}

Introducción: la obesidad es una enfermedad crónica multifactorial que genera repercusiones sistémicas; caracterizado por un Índice de Masa Corporal (IMC) superior a 30. El aislamiento social en la pandemia COVID-19 interfirió directamente en el tratamiento de pacientes obesos y enfermos antes sin comorbilidad. Metodología: Se trata de una revisión de literatura cualitativa, con búsqueda de estudios en las bases de datos de la Virtual Health Library (BVS), Google Scholar, PubMed y Scientific Library Online (SciELO). Para la investigación se utilizaron los descriptores COVID-19, aislamiento social, obesidad, salud mental y trastornos alimentarios. Se incluyeron publicaciones entre 2017 y 2021 en portugués e inglés disponibles en texto completo. Se seleccionaron 15 artículos de los 71 encontrados. Resultados y discusión: se advirtió que el aislamiento interfiere negativamente en el tratamiento de los pacientes obesos, ya que los lugares para el ejercicio físico fueron restringidos y el confinamiento domiciliario dificulta el tratamiento. Otro problema es el desarrollo de atracones, ya que los alimentos se utilizan para aliviar la ansiedad, la depresión y los problemas de estrés. Conclusión: COVID-19 provocó problemas que van más allá de los casos de infección por virus, ya que el aislamiento social afectó directamente el tratamiento de los pacientes obesos.

Palabras clave: COVID-19; Aislamiento social; Obesidad.

\section{Introdução}

A obesidade é indubitavelmente uma situação de epidemia em escala mundial. Segundo a Sociedade Brasileira de Endocrinologia e Metabologia (SBEM) (2020), a pessoa é considerada obesa quando possui um Índice de Massa Corporal (IMC) maior que 30 (trinta), sendo ele calculado pelo peso em quilogramas (kg) divido pela altura em metros (m), elevada ao quadrado $\left(\mathrm{IMC}=\mathrm{kg} / \mathrm{m}^{2}\right.$ ). A obesidade é uma doença multifatorial que envolve fatores biológicos, nutricionais, ambientais, sociais, culturais, econômicos e políticos, sendo caracterizada pelo acúmulo excessivo de tecido adiposo e alteração desfavorável na distribuição da adiposidade corporal (Porto e Porto, 2019).

As clássicas alterações metabólicas da obesidade associam-se tanto a um quadro infamatório crônico, devido a altos níveis de citocinas pró-inflamatórias, quanto a complicações prevalentes nos pacientes obesos, como resistência insulínica, hipertensão arterial sistêmica e dislipidemia. Ademais, cabe ressaltar os efeitos psicológicos negativos como quadros depressivos e transtornos de ansiedade decorrentes do estado de obesidade (Sbem, 2018; Sbem, 2020).

Em 2017 percebeu-se a gravidade do quadro epidemiológico da obesidade no mundo, visto que em 27 anos o IMC mundial aumentou $127 \%$, e no Brasil a situação também é considerada severa, pois a doença acomete mais da metade da população adulta, e dados da Pesquisa Nacional de Saúde de 2019, demonstraram 60\% da população brasileira maior de 18 anos tinha excesso de peso (IMC $\geq 25$ ), e, destes, $26 \%$ estavam obesos. E isto está justificado pela alta prevalência de maus hábitos alimentares e sedentarismo. Ademais a principal medida terapêutica em casos de obesidade leve é a mudança no estilo 
de vida, que envolve o hábito de dieta balanceada e atividade física regular; enquanto que nos casos mais extremos, pode ser indicado além do tratamento não farmacológico, a utilização de medicamentos e cirurgia bariátrica (Sbem, 2018; Boyd, 2019).

Nesse contexto, apesar de não existirem dados específicos, estima-se que a pandemia do COVID-19 e a implementação do distanciamento social sejam cofatores para a ascensão da quantidade de obesos por afetar diversas esferas do tratamento da obesidade (Ferrante et al., 2020). Sendo assim, esse trabalho tem por objetivo propor uma revisão de literatura, a fim de discutir os possíveis impactos decorrentes do isolamento social, sobre a obesidade, durante a pandemia de COVID-19.

\section{Metodologia}

O desenvolvimento textual deu-se por meio da elaboração de uma revisão bibliográfica de natureza qualitativa integrativa (Ludke, 2013). Os artigos para embasamento científico foram criteriosamente selecionados na Biblioteca Virtual em Saúde (BVS), Google Scholar, PubMed e Scientific Library Online (SciELO), escritos nas línguas inglesa e portuguesa. Os descritores utilizados para realização de buscas foram: COVID-19, isolamento social, obesidade, saúde mental e transtornos alimentares.

Foram escolhidos 15 artigos dentre 71 encontrados nos bancos de dados pesquisados. Os critérios para inclusão na revisão foram: artigos publicados no período entre 2017 e 2021 e relevância temática assegurada por meio da leitura prévia dos resumos de todos os artigos encontrados. Os critérios de exclusão consistem em artigos publicados antes de 2017, discrepância do conteúdo para a discussão proposta e impossibilidade de relacionar a temática do artigo com a situação de isolamento social provocada pela pandemia de COVID-19.

Outros materiais utilizados no projeto consistem em uma fonte atualizada de livro contendo aspectos clínicos e fisiopatológicos da obesidade, manuais relacionados ao tema disponibilizados pelo Ministério da Saúde e matérias publicadas na plataforma virtual da SBEM entre os anos de 2018 e 2020. Durante a pesquisa para coleta de informações, no banco de dados da SBEM, os descritores previamente explicitados foram novamente usados.

\section{Resultados e Discussão}

\subsection{Isolamento social como contribuinte para a obesidade}

A pandemia causada pelo novo coronavírus, tem gerado modificações estruturais significativas nas interações sociais, uma vez que a forma de prevenção mais efetiva e recomendada pelas autoridades em saúde para conter a disseminação do sarscov-2, é o distanciamento e isolamento social, vinculado à utilização de máscaras (Garcia, 2020; Schuchmann, et al, 2020).

Todavia, com o rápido avançar da infecção, diversos países adotaram o estado de lockdown, o qual permite apenas o funcionamento de atividades essenciais, que não incluem academias ou locais públicos para a prática de atividades físicas (Houvessou, et al, 2021).

Nesse contexto, o tratamento da obesidade, o qual deve ser realizado a partir da mudança do estilo de vida, como a prática de atividades físicas, foi dificultado pelo inacesso aos locais de realização de exercícios físicos. Ao relacionar a situação de isolamento com o tratamento para obesidade, percebe-se que este interfere de modo negativo na medida terapêutica, visto que as academias, que constituem o principal local de desenvolvimento físico para os pacientes obesos foram fechadas, e a reclusão em casa faz com que o tratamento seja abandonado (Pitanga, et al, 2020; Ministério da saúde, 2020).

Tal fato contribui para a evolução da obesidade, que além de desencadear doenças cardiovasculares, é importante fator de risco para a forma clínica grave da COVID-19, visto que o tecido adiposo, principalmente o visceral é uma intensa fonte de citocinas pró-inflamatórias, como o TNF- $\alpha$ e a IL-6. Ademais, o indivíduo em estado de obesidade possui alta 
concentração da enzima conversora de angiotensina 2 (ECA2), a qual constitui uma receptora para o Sars-Cov2 (Borges et al, 2021; Sattar et al, 2020).

\subsection{Alimentação em período de reclusão social}

No estudo de Durães, et al (2020), é ressaltado que o isolamento social contribui para a perda de bons hábitos alimentares, e o desenvolvimento de hábitos não saudáveis, como a alta ingestão calórica proveniente de alimentos ricos em carboidratos e lipídeos, como fastfood, frituras e doces. Nesse aspecto, outro ponto que se relaciona com os maus hábitos alimentares é o tédio, resultado da falta de atividades de lazer fora do ambiente do lar. Fato que pode desencadear transtornos alimentares, como a compulsão alimentar tanto em pessoas obesas, quanto em indivíduos sem a comorbidade que podem vir a desenvolver esta doença. Visto que o ato de se alimentar é um dos estímulos que ativam o sistema de recompensa do organismo, pois é um ato prazeroso, no qual o indivíduo sente alívio da tensão que atinge todo o globo (Karsburg, 2020; Touyz, 2020). Também, o estudo de Florêncio Júnior, et al (2020) foi convergente nesse sentido, visto que aponta que o isolamento contribui para o ganho de gordura.

\subsection{Situação de quarentena e as mudanças de rotina}

O isolamento social decorrente da situação de quarentena está ligado não somente à descontinuidade terapêutica da obesidade, como também ao aumento da incidência desta em pessoas antes saudáveis. Tal situação decorre da interação de aspectos psicossociais, como, a readaptação das formas laborais, já que muitas formas de trabalhos passaram a ser desenvolvidas no ambiente doméstico. Esse fenômeno, denominado de home office, contribui para a diminuição da vontade e do vigor para realização de atividades físicas além de aumentar o comodismo e o sedentarismo. Já os ambientes presencias de trabalho e espaços para prática de atividades físicas propiciam notadamente interações pessoais, que agem como estímulo para a continuidade do tratamento, como o apoio oferecido pelo educador físico. (Ministério da saúde, 2020; Pitanga et al, 2020).

\subsection{Fatores psíquicos do isolamento que interferem no tratamento da obesidade}

$\mathrm{O}$ aumento da desesperança e da infelicidade entre as pessoas que se encontram em situação de reclusão, foi relatado em estudos desenvolvidos durante a pandemia de COVID-19, e se relacionam com a perda da perspectiva de vida, e de foco nos projetos, podendo gerar e agravar distúrbios psíquicos como depressão, estresse e ansiedade. Nesse aspecto, tais transtornos diminuem a síntese e a liberação de hormônios e neurotransmissores que promovem a sensação de bem-estar, prazer e felicidade (Cunha, et al. 2020). Tal situação leva o indivíduo a buscar prazer na alimentação muitas vezes de forma compulsiva. Desse modo, a infecção pelo Sars-cov-2 afeta não somente os pulmões, mas o ser biopsicossocial de forma integral, desencadeando e agravando patologias, como no caso da obesidade (Razzoli, et al, 2017; Ministério da saúde, 2020).

\section{Considerações finais}

Com base nas informações contidas nas literaturas descritas, conclui-se que o isolamento social decorrente da pandemia do covid-19 interferiu negativamente tanto no tratamento dos indivíduos obesos ou com sobrepeso quanto causou um processo de adoecimento de pessoas anteriormente saudáveis. Processo esse causado por questões espaciais, como a impossibilidade da prática de exercícios físicos em qualquer ambiente com interações sociais físicas (o que estimularia os praticantes a manter a rotina). Além disso, questões psicológicas como ansiedade, estresse e depressão favoreceram uma compulsão alimentar, pois a comida é utilizada para suprir o prazer antes oferecido pelos hormônios como serotonina e dopamina. Logo, é indiscutível que a pandemia pelo novo coronavírus influencia esferas que vão muito além da infecção pelos 
SARS-CoV-2 e são indispensáveis mais trabalhos sobre essa temática, principalmente com as consequências que a sociedade pode enfrentar, uma vez que a obesidade é um problema de saúde pública que precisa de atenção e cuidado.

\section{Referências}

Borges, J. F. T., Rebelo, A. A. F., Spinasse, G. S., dos Santos Neto, J. R., Massoud, A. B. P., de Miranda, G. F., \& de Mendonça, M. H. R. (2021). A obesidade como fator de risco no pior prognóstico do Covid-19: uma revisão integrativa. Brazilian Journal of Health Review, 4(1), $3699-3712$.

Costa, B. M. D., Neves, D. K. O. D., Meloni, L. C., Cordeiro, S. A., Lima, T. P. B., Marinho, W. N., \& Santos, M. (2020). Guia de orientações em relação à alimentação e exercício físico: Diante da pandemia da doença pelo SARS-CoV-2 (COVID-19).

Cunha, D. S., Pardo, D. S. V., Caputo, L. R. L. G., \& Rabelo, E. M. (2020) Relação da obesidade com a depressão em adultos. Revista Científica Online. $12(2)$.

de Aro, F., Pereira, B. V., Navarro, D., \& Bernardo, A. (2021). Comportamento alimentar em tempos de pandemia por Covid-19. Brazilian Journal of Development, 7(6), 59736-59748.

Durães, S. A., Souza, T. S., Gome, Y. A. R., \& de Pinho, L. (2020). Implicações da pandemia da covid-19 nos hábitos alimentares. Revista Unimontes Científica, 22(2), 1-20.

Ferrante, G., Camussi, E., Piccinelli, C., Senore, C., Armaroli, P., Ortale, A., \& Giordano, L. (2020). Did social isolation during the SARS-CoV-2 epidemic have an impact on the lifestyles of citizens?. Epidemiol Prev, 353-362.

Garcia, L. P. (2020). Uso de máscara facial para limitar a transmissão da COVID-19. Epidemiologia e Serviços de Saúde, 29 , e2020023.

Houvèssou, G. M., Souza, T. P. D., \& Silveira, M. F. D. (2021). Medidas de contenção de tipo lockdown para prevenção e controle da COVID-19: estudo ecológico descritivo, com dados da África do Sul, Alemanha, Brasil, Espanha, Estados Unidos, Itália e Nova Zelândia, fevereiro a agosto de 2020. Epidemiologia e Serviços de Saúde, 30.

Júnior, P. G. F., Paiano, R., \& dos Santos Costa, A. (2020). Isolamento social: consequências físicas e mentais da inatividade física em crianças e adolescentes. Revista Brasileira de Atividade Física \& Saúde, 25, 1-2.

Ludke, M., \& André, M. (2013). Pesquisa em educação: abordagens qualitativas. Em Aberto, 5(31).

Ministério da saúde (2018). Manual - Como organizar o cuidado de pessoas com doenças crônicas na APS no contexto da pandemia. Secretaria de Atenção Primária à Saúde.

Pitanga, F. J. G., Beck, C. C., \& Pitanga, C. P. S. (2020). Inatividade física, obesidade e COVID-19: perspectivas entre múltiplas pandemias. Revista Brasileira de Atividade Física \& Saúde, 25, 1-4.

Porto, C. C., \& Porto, A. L. (2019). Semiologia Médica. Guanabara Koogan

Razzoli, M., Pearson, C., Crow, S., \& Bartolomucci, A. (2017). Stress, overeating, and obesity: Insights from human studies and preclinical models. Neuroscience \& Biobehavioral Reviews, 76, 154-162.

Sattar, N., McInnes, I. B., \& McMurray, J. J. (2020). Obesity is a risk factor for severe COVID-19 infection: multiple potential mechanisms. Circulation, 142(1), 4-6.

Schuchmann, A. Z., Schnorrenberger, B. L., Chiquetti, M. E., Gaiki, R. S., Raimann, B. W., \& Maeyama, M. A. (2020). Isolamento social vertical X Isolamento social horizontal: os dilemas sanitários e sociais no enfrentamento da pandemia de COVID-19. Brazilian Journal of Health Review, 3(2), 35563576.

Sociedade brasileira de endocrinologia e metabologia (2018). 10 Coisas que Você Precisa Saber Sobre Obesidade. endocrino.org.br/10-coisas-que-voceprecisa-saber-sobre-obesidade/

Sociedade brasileira de endocrinologia e metabologia (2018). IBGE: Obesidade Mais que Dobra no País. endocrino.org.br/ibge-obesidade-mais-que-dobra-nopais/

Sociedade brasileira de endocrinologia e metabologia (2018). Obesidade e os Riscos do Covid-19. endocrino.org.br/obesidade-e-os-riscos-do-covid-19/

Swinburn, B. A., Kraak, V. I., Allender, S., Atkins, V. J., Baker, P. I., Bogard, J. R., \& Dietz, W. H. (2019). The global syndemic of obesity, undernutrition, and climate change: the Lancet Commission report. The lancet, 393(10173), 791-846.

Touyz, S., Lacey, H., \& Hay, P. (2020). Eating disorders in the time of COVID-19. 